\title{
Research Paper: Effectiveness of Chest Physiotherapy in Cerebrovascular Accident Patients With Aspiration Pneumonia
}

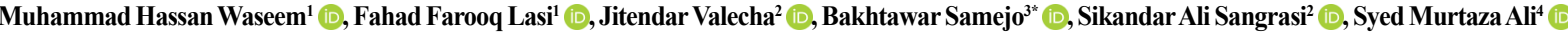

1. Department of Physiotherapy, Karachi Institute of Physiotherapy and Rehabilitation Sciences (KIPRS), Karachi Sindh, Pakistan.

2. Department of Physiotherapy, Institute of Physiotherapy and Rehabilitation Sciences, LUMHS, Jamshoro, Pakistan.

3. Department of Physiotherapy, Momal Institute of Physiotherapy and Allied Health Sciences, Hyderabad Sindh, Pakistan.

4. Department of Physiotherapy, Institute of Physiotherapy and Rehabilitation Sciences, PUMHS, Nawabshah, Pakistan.

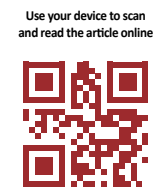

Citationt Waseem MH, Farooq Lasi F, Valecha J, Samejo B, Sangrasi SA, Ali SM. Effectiveness of Chest Physiotherapy in Cerebrovascular Accident Patients With Aspiration Pneumonia. Journal of Modern Rehabilitation. 2020; 15(1):47-52. http:// dx.doi.org/10.32598/JMR.15.1.7

http://dx.doi.org/10.32598/JMR.15.1.7

Article info:

Received: 08 Sep 2020

Accepted: 05 Oct 2020

Available Online: 01 Jan 2021

\section{Keywords:}

Stroke, Aspiration pneumonia, Chest physiotherapy,

Cerebrovascular accident

(CVA)

\section{A B S T RACT}

Introduction: According to $\mathrm{WHO}$, a stroke refers to rapidly developing clinical signs of focal (or global) disturbance of cerebral functions, with symptoms lasting 24 hours or longer or leading to death. The damage caused by a stroke can interrupt your normal swallowing and food or fluid is entered into your airways and lungs. Dysphagia can damage the lungs, which can trigger a lung infection (pneumonia). To assess the efficacy of chest physiotherapy in the prevention of aspiration pneumonia in stroke patients.

Materials and Methods: A quasi-experimental study was conducted with a sample size of 35 participants from C1 ward Agha Khan Hospital in Karachi City, Pakistan. The participants were selected via random sampling method. The inclusion criteria include patients of the $\mathrm{Cl}$ ward with a cerebrovascular accident, both male and female gender, with the age ranges from 45 to 63 years. All statistical analysis was done by using SPSS v. 19. The paired t test was used to evaluate the effectiveness of chest physiotherapy in a patient with aspiration pneumonia. The chest physiotherapy includes turning, postural drainage, percussion, vibration, deep breathing exercises, coughing, and suctioning were performed on a patient with aspiration pneumonia.

Results: The results of this study show pre-treatment and post-treatment chest congestion, heart rate, and respiratory rate show that the $\mathrm{P}$ value is highly significant.

Conclusion: This study concludes that chest physiotherapy seems to be effective in treating aspiration pneumonia in cerebrovascular accident patients. 


\section{Introduction}

A

ccording to WHO, a stroke refers to rapidly developing clinical signs of focal (or global) disturbance of cerebral function, with symptoms lasting 24 hours or longer or leading to death, with no apparent cause other than of vascular origin [1]. A stroke may either be ischemic or hemorrhagic disturbances of the cerebral blood circulation. Many risk factors have been described for stroke. They may refer to inherent biological traits such as age and sex; physiological characteristics that predict future occurrences such as high blood pressure, serum cholesterol, fibrinogen; behaviors such as smoking, diet, alcohol consumption, physical inactivity, social characteristics such as education, social class, and ethnicity and environmental factors that may be physical (temperature, altitude), geographical, or psychosocial [2]. The damage caused by a stroke can interrupt your normal swallowing or gag reflex and makes it possible for small particles of food or fluid to be inhaled into the airways and lungs instead of being swallowed and proceeding down your esophagus into your stomach [3]. Dysphagia can damage the lungs, which can trigger a lung infection (pneumonia) [4].

Several pulmonary syndromes are evident after aspiration based on the aspired material and its nature, the frequency, and the response of the host to the aspirated matter. The elderly population is at increased risk for pulmonary syndromes due to the high risk and incidence of dysphagia and gastroesophageal reflux. The feeding via the tube is usually preferred in the patients who continue to aspirate diluted food even after following several strategies; and in patients with stroke, the swallowing dysfunction ranges from $40 \%$ to $70 \%$ [5]. One of the important aspects of bronchial hygiene is Chest Physiotherapy (CPT) that can include rotation, percussion, postural drainage, vibration, and targeted cough techniques called directed cough. Some or all of the strategies can be used in conjunction with aerosol treatment regimens (bronchodilators or mucolytics) [6].

The objective of CPT is to channel bronchial fluid to the main airway with the help of gravity, external chest compressions, and removing fluid by coughing or catheter aspiration. Betterment in bronchial secretions mobilization helps in advanced ventilation-perfusion matching and normalizing functional residual capacity [7]. One of the studies focused on the effectiveness of chest physiotherapy as an adjunct treatment in children with pneumonia, 255 children between the age of 29 days and 12 years diagnosed with pneumonia were studied and it was found that respiratory rate and peripheral oxygen saturation were improved [8].

Another study provided a comprehensive overview and evidence to support the role of physiotherapy in the management of individuals with Cystic Fibrosis (CF), including airway clearance, exercise, and musculoskeletal concerns which can affect activities of daily living and respiratory health. They found that the role of physiotherapy in CF was complex and included airway clearance, exercise, and management of the long-term sequelae of musculoskeletal issues [9]. One of the studies focused on Pulmonary Rehabilitation (PR) in patients with Chronic Obstructive Lung Disease (COPD) to have an impact on health-related quality of life, exercise capacity, and improved dyspnea. The core components of the PR regime includes focused training and education of the muscle and chest physical therapy [10].

Several studies highlight the point regarding community-acquired pneumonia that generally because of aspiration and accounts for $5 \%-15 \%$ of the cases [11]. The mortality in neurological disorders results from dysphagia because of aspiration pneumonia. In the patients with neurologic dysphagia, gastroesophageal junction problems, or anatomical abnormalities of the upper airway tract are at high risk for oropharyngeal aspiration [6]. This study focused on Pulmonary Rehabilitation (PR) to treat dyspnea-related issues and improvement in the quality of life in patients with Chronic Obstructive Pulmonary Disease (COPD). The fundamental components of PR programs are muscle training, education, and chest physiotherapy [12]. Some literature discussed the effectiveness of chest physiotherapy and their results contradicted the already available results on aspiration pneumonia in stroke patients. Therefore, this study aims to assess the efficacy of chest physical therapy in the prevention of aspiration pneumonia.

\section{Materials and Methods}

A quasi-experimental study was conducted with a sample size of 35 participants from C1 ward Agha Khan Hospital, Karachi City, Pakistan. The participants were selected via random sampling method. The inclusion criteria include patients of the $\mathrm{C} 1$ ward diagnosed with a cerebrovascular accident, both male and female gender, within the age range of 45-63 years. All outpatients and those who were not diagnosed with the cerebrovascular accident were excluded from the study. All statistical analysis was done by using SPSS v. 19. The paired t test was used to evaluate the effectiveness of chest physiotherapy in patients with aspiration pneumonia. 


\section{Procedure}

\section{Turning}

Turning is changing the body's position over its long axis. The turning is used in conjunction with techniques designed to help the patient comfort and skin condition. However, attention towards chest precise position is necessary to affect fluid mobilization. Special beds that are adjusted periodically provide a combination of patient responses by hand.

\section{Postural drainage}

Postural drainage is the placement of the subject and bed in such a way that the carina is lower to a lung segment that needs to be drained. The direction of the lungs is almost as close to the ground as possible in a perpendicular position. The purpose is to move the liquid from the edges to the central airways to eliminate it. The average time is 3 to 15 minutes per section depending on the secretions properties.

\section{Percussion}

Percussion is also known as clapping and cupping. These terms refer to the rhythmic manual striking on the thoracic region on a lung segment that is being drained. The transmitted energy through the thoracic wall to the lung dislodges the adhered secretions on the lung tissue. Different devices (mechanical and pneumatic) can also be used for the same function. Percussion can also be used to initiate cough response by performing on large airways.

\section{Vibration}

Vibration is the repeated movements of hands placed along the rib cage directed towards expiratory chest movement. Slow rapid vibration (trembling) and slight pressure are used during breathing to emphasize this phase of the respiratory cycle during exhalation. The procedure mimics the forced airflow of a cough. The most powerful form of vibration manually in combination with positive pressure ventilation is called "artificial cough." This is used as an aid in removing sputum from disabled patients on ventilators. The mechanical instruments used to make vibrations are different from the manual method in which the active machine is used continuously during inhalation and exhalation.

\section{Deep breathing exercises}

It is used to fill the lungs with air and to ventilate all the areas.

\section{Coughing}

Coughing helps to separate fluid from the lungs so that mucus can be excreted or suctioned out. Patients remain upright and breathe deeply by their noses. Then they let out air cough or short puffs. The procedure is repeated several times a day.

\section{Suctioning}

The patient's airway clearance is an important procedure to mobilize secretions by tracheal suctioning. The procedure helps to remove the excess secretions and initiate cough reflex to maintain a clear airway. The suctioning is done by placing a catheter at the right spot that is approximately proximal to the secretion.

\section{Results}

SPSS v. 19 was used to analyze the data. The paired sample $t$ test was used for the comparison of mean differences between pre- and post-treatment. A P value of less than 0.05 was considered significant. Table 1 shows the mean and standard deviations of paired sample statistics as pair 1 pre-treatment chest congestion have std deviation 0.000 and mean as 0.000 while post-treatment chest congestion has std. deviation 0.482 and mean as 0.081 , it also shows pair 2 pre-treatment heart rate having std. deviation 0.382 and mean 0.065 while posttreatment heart rate has std. deviation 0.471 and mean 0.080 . Table 1 also shows pair 3 pre-treatment respiratory rate having std. deviation 0.355 and mean 0.060 while post-treatment respiratory rate has std. deviation 0.458 and mean 0.077 .

Table 2 shows paired samples correlation as pair 1 preand post-treatment chest congestion have 0.258 correlation, pair 2 pre-, and post-treatment heart rate has -0.182 correlation while pair 3 pre and post-treatment respiratory rate have 0.258 correlation.

Table 3 presents the $\mathrm{P}$ value of pre-treatment and posttreatment chest congestion, heart rate, and respiratory rate and the results show they are highly significant as the $\mathrm{P}$ value of chest congestion, heart rate, and respiratory rate after the chest physiotherapy was 0.000 that is much less than 0.005 .

\section{Discussion}

The present study results indicate that pulmonary rehabilitation has a positive outcome on inpatient aspirated lungs, the heart, respiratory rate, and quality of life. It 
Table 1. Mean $\pm S D$ of pre- and post-chest physiotherapy treatment $(n=35)$

\begin{tabular}{cccc}
\hline & Stage & Mean \pm SD & SEM \\
\hline \multirow{2}{*}{ Pair 1 } & Pre-treatment chest congestion & $2.00 \pm 0.000$ & 0.000 \\
& & $1.34 \pm 0.482$ & 0.081 \\
Pair 2 & Pre-treatment heart rate & $1.83 \pm 0.382$ & 0.065 \\
& & $1.31 \pm 0.471$ & 0.080 \\
Pair 3 & Pre-treatment respiratory rate & $1.86 \pm 0.355$ & 0.060 \\
\hline
\end{tabular}

Table 2. Correlation and significance of pre- and post-chest physiotherapy treatment $(n=35)$

\begin{tabular}{|c|c|c|c|}
\hline & Stage & Correlation & Sig. \\
\hline Pair 1 & $\begin{array}{l}\text { Pre-treatment chest congestion } \\
\text { Post-treatment chest congestion }\end{array}$ & 0.258 & 0.134 \\
\hline Pair 2 & $\begin{array}{l}\text { Pre-treatment heart rate } \\
\text { Post-treatment heart rate }\end{array}$ & -0.182 & 0.295 \\
\hline Pair 3 & $\begin{array}{l}\text { Pre-treatment respiratory rate } \\
\text { Post-treatment respiratory rate }\end{array}$ & 0.258 & 0.134 \\
\hline
\end{tabular}

Paired samples correlations

$J \mathrm{MR}$

has been shown that short-term inpatient PR programs bring out positive results similar to long-term programs, and results in a short length of hospitalization. Our study results showed improved pulmonary compliance by reducing crackles in the lungs and improved tachycardia cretion, and weak cough are the main symptoms restricting the improvement in stroke patients. Aspiration pneumonia is very common in stroke patients due to the weak or absence of gage reflex. and tachypnea. Aspiration pneumonia, absent gage, se-

Table 3. P value (significance) of pre- and post-chest physiotherapy treatment

\begin{tabular}{|c|c|c|c|c|c|c|c|c|}
\hline & \multirow{3}{*}{ Stage } & \multicolumn{4}{|c|}{ Paired Differences } & \multirow{3}{*}{$\mathbf{t}$} & \multirow{3}{*}{ df } & \multirow{3}{*}{ Sig. (2-Tailed) } \\
\hline & & \multirow{2}{*}{ Mean士SD } & \multirow{2}{*}{ SEM } & \multicolumn{2}{|c|}{$\begin{array}{l}\text { 95\% Confidence Inter- } \\
\text { val of Difference }\end{array}$} & & & \\
\hline & & & & Lowe & Upper & & & \\
\hline Pair 1 & $\begin{array}{l}\text { Pre-treatment chest congestion } \\
\text { Post-treatment chest congestion }\end{array}$ & $0.657 \pm 0.482$ & 0.081 & 0.492 & 0.823 & 8.073 & 34 & 0.000 \\
\hline Pair 2 & $\begin{array}{l}\text { Pre-treatment heart rate } \\
\text { Post-treatment heart rate }\end{array}$ & $0.514 \pm 0.658$ & 0.111 & 0.288 & 0.740 & 4.620 & 34 & 0.000 \\
\hline Pair 3 & $\begin{array}{l}\text { Pre-treatment respiratory rate } \\
\text { Post-treatment respiratory rate }\end{array}$ & $0.571 \pm 0.502$ & 0.085 & 0.399 & 0.744 & 6.733 & 34 & 0.000 \\
\hline
\end{tabular}

Paired samples Test 
This study revealed that the patients receiving chest physiotherapy showed a marked decrease of crackles in the lungs. This study also showed a remarkable effect of chest physiotherapy on heart rate and respiratory rate. Most patients had aspirated, had severe chest congestion, and decreased pulmonary compliance but when chest physiotherapy was given to them. Then, chest congestion in those patients was improved. In some cases, there was a mild improvement and in other cases, there was moderate to high degree improvement.

Some clinical studies have controversial findings in case of pneumonia for chest physiotherapy effectiveness and concluded that resolution of pneumonia was not useful and large multicenter trials are needed to further investigate the results [13]. Another study was titled chest physiotherapy in primary pneumonia with a sample size of 171 patients focusing on single-blind trial, placebo trial, and random allocation. Besides, postural drainage, external help with breathing, percussion, and vibration and advice expectoration to avoid thrombosis. The principles of pharmaceutical management were the same in the two groups concluded that chest physiotherapy had beneficial effects in patients with pulmonary infection [14].

One of the studies on randomized control trial of chest physical therapy for hospitalized children unable to ensure that chest physical therapy as a supplement to standard treatment accelerates the resolution of clinical symptoms of children with acute pneumonia in-hospital care. Clinical resolution time and length of hospital stay were the same for the intervention and control groups. Besides, this study showed that patients receiving pulmonary physical therapy have a longer cough and rhonchi in lung auscultation. However, persistence in these respiratory signs and symptoms may not represent an undesirable clinical manifestation. Coughing might be induced by a physical therapist which is an important part of chest physical therapy. Rhonchus, a typical "secretion sound" in lung sounds, might be extended by chest physical therapy as it will dislodge tracheobronchial secretions and produce this sound [15].

However, several studies focusing on the role of physiotherapy in preventing post-extubation atelectasis are not in favor of our finding [16]. On the other hand, a comparison by Deakins and Chatburn on intrapulmonary percussive ventilation along with traditional chest physiotherapy to identify their effects on improving atelectasis in children. It was found that clinically important improvement in atelectasis was significantly higher in patients who have undergone intrapulmonary percussive ventilation therapy as compared to chest physiotherapy [17].

\section{Study limitation}

The study had some limitations which are namely lack of control group, small sample size, and only one hospital used for data collection. Some other factors like large sample size, long-term effects, detailed clinical studies, medication, psychological and cost assessments are needed to confirm these results that might change our results.

\section{Implications of the study}

Chest physiotherapy plays a vital role in the reduction of ICU time and hospital stay. The CVA patients have difficulty in airway clearance thus chest physiotherapy helps to clear the airway and increase the chest efficiency and helps in improving saturation and arterial blood gases. For airway clearance in pneumonia, the patient's chest physiotherapy plays a vital role in reducing the drug intake for clearing the obstruction. These findings are helpful for all the clinicians and thus help to reduce the drug intake and adding complications. The results may be useful for further research.

\section{Conclusion}

This study concludes that chest physiotherapy seems to favor aspiration pneumonia in CVA patients. With a large number of patients and more randomized controlled trials to evaluate the role of therapeutic chest physiotherapy in CVA patients with aspiration pneumonia and its adverse effects are needed to add more literature to the scientific society.

\section{Ethical Considerations}

\section{Compliance with ethical guidelines}

All participants were assured of their confidentiality, written informed consent was taken before participation. The study was approved by the Ethics Review Committee (KIPRS/R\&D/ERC/2020-01).

\section{Funding}

This research did not receive any specific grant from funding agencies in the public, commercial, or non-profit sectors.

\section{Authors contributions}

Conceptualization, research design: Muhammad Hassan Waseem, Fahad Farooq Lasi, JitendarValecha, Bakhtawar Samejo; Data collection, assembly, analysis, interpretation, writing the article, final approval of the 
article: All authors; Critical revision of the article: Fahad Farooq Lasi, Muhammad Hassan Waseem.

\section{Conflict of interest}

All authors declared no conflict of interest.

\section{Acknowledgements}

The authors would like to thank to the Ethics Review Committee of Karachi Institute of Physiotherapy and Rehabilitation Sciences.

\section{References}

[1] WHO MONICA Project Principal Investigators. The World Health Organization MONICA project (monitoring trends and determinants in cardiovascular disease): A major international collabaration. Journal of Clinical Epidemiology Oxford. 1988; 41(2):105-14. [DOI:10.1016/0895-4356(88)90084-4]

[2] Della-Morte D, Guadagni F, Palmirotta R, Testa G, Caso V, Paciaroni M, et al. Genetics of ischemic stroke, stroke-related risk factors, stroke precursors and treatments. Pharmacogenomics. 2012; 13(5):595-613. [DOI:10.2217/pgs.12.14] [PMID]

[3] Bonita R, Duncan J, Truelsen T, Jackson RT, Beaglehole $R$. Passive smoking as well as active smoking increases the risk of acute stroke. Tobacco Control. 1999; 8(2):156-60. [DOI:10.1136/tc.8.2.156] [PMID] [PMCID]

[4] O'donnell MJ, Xavier D, Liu L, Zhang H, Chin SL, RaoMelacini $\mathrm{P}$, et al. Risk factors for ischaemic and intracerebral haemorrhagic stroke in 22 countries (the INTERSTROKE study): A case-control study. The Lancet. 2010; 376(9735):11223. [DOI:10.1016/S0140-6736(10)60834-3]

[5] Ebihara S, Sekiya H, Miyagi M, Ebihara T, Okazaki T. Dysphagia, dystussia, and aspiration pneumonia in elderly people. Journal of Thoracic Disease. 2016; 8(3):632-9. [DOI:10.21037/jtd.2016.02.60] [PMID] [PMCID]

[6] Stein MR. Gastroesophageal reflux disease and airway disease. $1^{\text {th }}$ ed. London: Informa Healthcare; 1999. https:/ / www. amazon.com/Gastroesophageal-Reflux-Disease-Airway-Biology/dp/0824702301

[7] Marik PE, Kaplan D. Aspiration pneumonia and dysphagia in the elderly. Chest. 2003; 124(1):328-36. [DOI:10.1378/ chest.124.1.328] [PMID]

[8] Yoneyama T, Yoshida M, Matsui T, Sasaki H. Oral care and pneumonia. The Lancet. 1999; 354(9177):515. [DOI:10.1016/ S0140-6736(05)75550-1]

[9] Mann G, Hankey GJ, Cameron D. Swallowing function after stroke: prognosis and prognostic factors at 6 months. Stroke. 1999; 30(4):744-8. [DOI:10.1161/01.STR.30.4.744] [PMID]

[10] Perren A, Zürcher P, Schefold JC. Clinical approaches to assess post-extubation dysphagia (PED) in the critically ill. Dysphagia. 2019; 34(4):475-86. [DOI:10.1007/s00455-01909977-w] [PMID]
[11] Torres A, Serra-Batlles J, Ferrer A, Jiménez P, Celis R, Cobo E, et al. Severe community-acquired pneumonia. The American Review of Respiratory Disease. 1991; 144(2):312-8. [DOI:10.1164/ajrccm/144.2.312] [PMID]

[12] Ney DM, Weiss JM, Kind AJH, Robbins J. Senescent swallowing: impact, strategies, and interventions. Nutrition in Clinical Practice. 2009; 24(3):395-413. [DOI:10.1177/0884533609332005] [PMID] [PMCID]

[13] Peruzzi WT, Smith B. Bronchial hygiene therapy. Critical Care Clinics. 1995; 11(1):79-96. [DOI:10.1016/S07490704(18)30086-1]

[14] Britton S, Bejstedt M, Vedin L. Chest physiotherapy in primary pneumonia. British Medical Journal (Clinical Research Ed.). 1985; 290(6483):1703-4. [DOI:10.1136/bmj.290.6483.1703] [PMID] [PMCID]

[15] Paludo C, Zhang L, Lincho CS, Lemos DV, Real GG, Bergamin JA. Chest physical therapy for children hospitalised with acute pneumonia: A randomised controlled trial. Thorax. 2008; 63(9):791-4. [DOI:10.1136/thx.2007.088195] [PMID]

[16] Bilan N, Poorshiri B. The role of chest physiotherapy in prevention of postextubation atelectasis in pediatric patients with neuromuscular diseases. Iranian Journal of Child Neurology. 2013;7(1):21-4. [PMCID]

[17] Yen Ha TK, Bui TD, Tran AT, Badin P, Toussaint M, Nguyen AT. Atelectatic children treated with intrapulmonary percussive ventilation via a face mask: Clinical trial and literature overview. Pediatrics International. 2007; 49(4):502-7. [DOI:10.1111/j.1442-200X.2007.02385.x] [PMID] 